\title{
Causes and Countermeasures of Abnormal Tones of Once-Through Boiler
}

\author{
Kunihiko Ishihara
}

\begin{abstract}
This paper describes the causes of abnormal tones generated from the small boiler and the countermeasures to suppress it. The abnormal tones caused by the acoustic resonance or the self-excited mechanism have ever been experienced. The baffle plate has been well used to suppress such kinds of tones. This countermeasure, however, can't be applied to the present boiler (called once- through boiler) because of the flow directional resonant mode. Then, the wall with holes was used to weaken the vortex strength which caused the abnormal tones. As a result, the abnormal tones disappeared completely.
\end{abstract}

Index Terms - Aerodynamic acoustics, Once-through boiler, Resonance, Self-sustained tone, Plate with holes

\section{INTRODUCTION}

In heat exchangers, such as boilers and gas heaters, tube banks are set in a duct and the water passes through inside the tube and warm gases outside the tube. When the flow velocity increases Karman vortex shedding occurs behind the tubes.

The vortex shedding frequency is proportional to the flow velocity. On the other hand, the duct has an acoustic natural frequency determined by the duct size and the sound speed. The acoustic natural frequency is independent of the flow velocity. A resonance phenomenon occurs at a certain velocity when the vortex shedding frequency coincides with the acoustic natural frequency [1-15].

An once-through boiler is widely used due to because small, quick start and high efficiency. In ordinary, at the heat conducting unit of a water pipe type large boiler, it is well known that an air column resonance occurs in the space constructed with both duct walls.

However, the air column resonance also occurs in a special boiler like a multi-tube type once-through boiler but it is different with that of ordinary water pipe type boiler. The countermeasure has been staying in empirical method because of the actual situation being unknown.

This boiler is a general purpose small boiler made into a series $350 \mathrm{~kg} / \mathrm{h}$ to $6000 \mathrm{~kg} / \mathrm{h}$ of evaporation amount. The construction is shown in Fig.1. The tubes line up in two rows and tubes are connected with fin plates. Inside of inner tube rows makes up a furnace. Combustion gases flow into the inner tubes row at a part and enter the space after dividing into two directions and meet after half a lapping. It gets out of the chimney.

As described below, this resonance is easily presumed to be the air column vibration at the boundary condition of both ends being open but self-sustained mechanism has not been known yet.

Kunihiko Ishihara, Department of Health and Welfare, Tokushima Bunri University, Shido, Sanuki-city, Kagawa, Japan, +81878945111.
In this paper, at first the measurement will be performed to know the relation between the operation conditions and the state of noise, next the cause will be presumed from the measured data and from the findings obtained from the results of some countermeasures, the cause is found out inversely, Finally the cause will be determined by some analyses.

\section{SURVEY IN BOILER}

\section{A. Specification of Real Boiler}

Fig.1 shows the outline of the boiler used in the present survey. This boiler is constructed with the combustion chamber and the convective heat transfer (Combustion gas flow path). Water tubes of inner and outer circumference 2 rows are combined at equal intervals circumferential direction through the plate and the gas flow path is formed. High temperature combustion gases enter in two flow routes $\mathrm{A}$ and $\mathrm{B}$ and discharge at exit port after meeting. During that it changes the water in the tube to the vapor. Therefore half of the exit flow velocity is the velocity in the circular flow path and given by $V_{\mathrm{g}}=(9 / 5) \times V$.
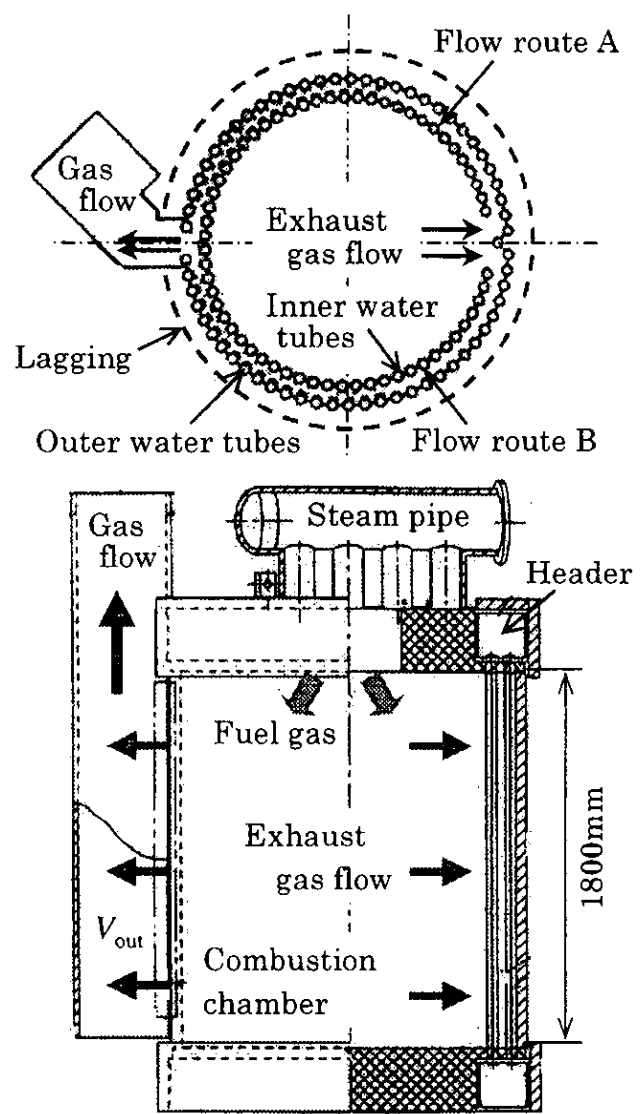

Fig.1 Outline of real boiler 


\section{B. Experimental Method}

In order to grasp the noise status, the exit velocity is measured by the anemometer and the sound pressure level at $1 \mathrm{~m}$ apart from the real boiler is also measured by the microphone when the damper opening becomes larger gradually. The signals recorded is frequency analyzed by a FFT analyzer (RION SA-78). The frequency range is $0 \mathrm{~Hz}$ to $1000 \mathrm{~Hz}$.

Abnormal tones in question are the phenomena independent of the heat because it occurs just let the air flow. Then the experiment in cold state was carried out here.

The air flow rate is changed by varying opening of the damper. Fig. 2 shows spectra of the sound pressure level and we can see the frequency of the peak level for the exit flow velocity $V_{\text {out }}$.

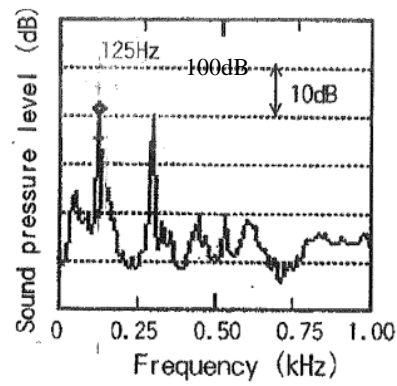

(a) $V_{\text {out }} \doteqdot 15 \mathrm{~m} / \mathrm{s}$

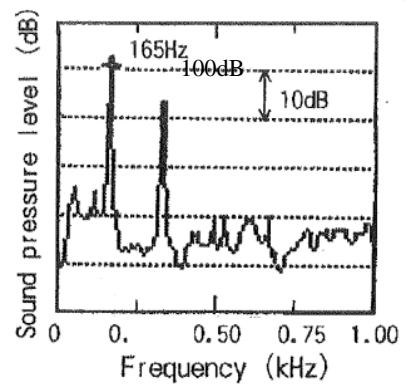

(b) $V_{\text {out }} \fallingdotseq 18 \mathrm{~m} / \mathrm{s}$
Fig.2 Peak SPL and its frequency to wind velocity

As can be seen in Fig.2, the sound pressure level of $80 \mathrm{~dB}$ could be measured below $13 \mathrm{~m} / \mathrm{s}$ where I could not feel the abnormal tone. at $14 \mathrm{~m} / \mathrm{s}$ and above, the pure tone of $92 \mathrm{~dB}$ could be felt and it continued until $17 \mathrm{~m} / \mathrm{s}$. Beyond it the pure tone of $100 \mathrm{~dB}$ and $165 \mathrm{~Hz}$ occurred next. During this the sound pressure level did not go down.

We measured the acoustic modes of $125 \mathrm{~Hz}$ and $165 \mathrm{~Hz}$ by traversing the microphone outside of the boiler. As a result, we obtained two modes as shown in Fig.3. They were (1) Combined mode of circumferential mode of $\lambda=L_{\mathrm{c}}$ and axial mode of $\lambda / 2=L_{\mathrm{a}}$, which frequency is $125 \mathrm{~Hz}$, (2) Circumferential mode of $\lambda=L_{\mathrm{c}} / 2$, which frequency is $160 \mathrm{~Hz}$. Where $L_{\mathrm{c}}$ and $L_{\mathrm{a}}$ are the circumferential length and the axial length of the boiler respectively. That is to say, $L_{\mathrm{c}}=4.52 \mathrm{~m}$ and $L_{\mathrm{a}}=1.8 \mathrm{~m}$.

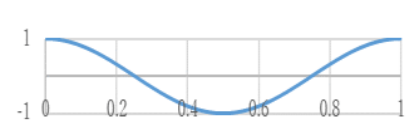

circumferential

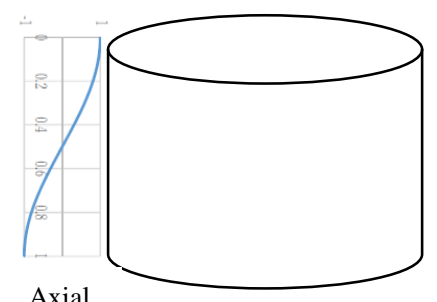

Axial

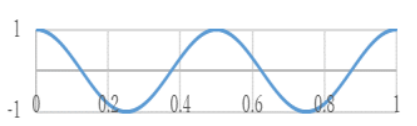

Circumferential

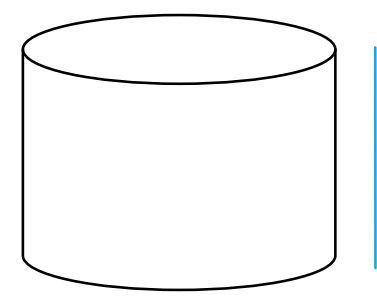

Axial
Fig.3 Resonant modes of real boiler
As the acoustic field constructed by inner and outer tubes is equivalent to that of a rectangular parallelepiped, these frequencies can be obtained by the equations.

The natural acoustic frequency of $\lambda=L_{\mathrm{c}}$ :

$f_{1}=\mathrm{c} / L_{\mathrm{c}}=340 / 4.525=75 \mathrm{~Hz}$

The natural acoustic frequency of $\lambda=L_{\mathrm{c}} / 2$ :

$f_{2}=\mathrm{c} /\left(L_{\mathrm{c}} / 2\right)=340 / 2.263=150 \mathrm{~Hz}$

The natural acoustic frequency of $\lambda=2 L_{\mathrm{a}}$ $f_{3}=\mathrm{c} / 2 \quad L_{\mathrm{a}}=340 / 2 / 1.8=95 \mathrm{~Hz}$

From these results, the mode of $125 \mathrm{~Hz}$ is the combined mode of $f_{1}$ and $f_{3}$. Then

$$
f_{13}=\sqrt{f_{1}^{2}+f_{3}^{2}}=121 \mathrm{~Hz}
$$

This value almost coincided with the measured value $125 \mathrm{~Hz}$. Therefore, the corresponding of natural acoustic frequencies and modes are believed to be correct.

From above examination, it was confirmed that the abnormal tones had natural acoustic frequencies of the acoustic field constructed with inner and outer tubes. What excites the acoustic field is easily imagined to be the vortices. Then we presumed that this phenomenon was the resonance or the lock-in phenomenon. We will show the evidences in the next Chapter.

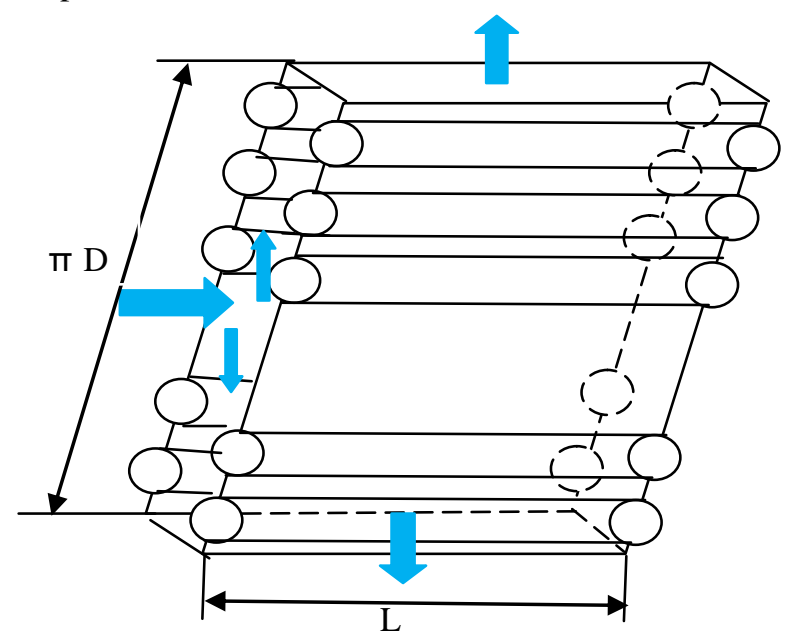

$\mathrm{L}=1.8 \mathrm{~m}, \mathrm{D}=1.44 \mathrm{~m}, \mathrm{~L}^{\prime}=\pi \mathrm{D}=4.52 \mathrm{~m}$

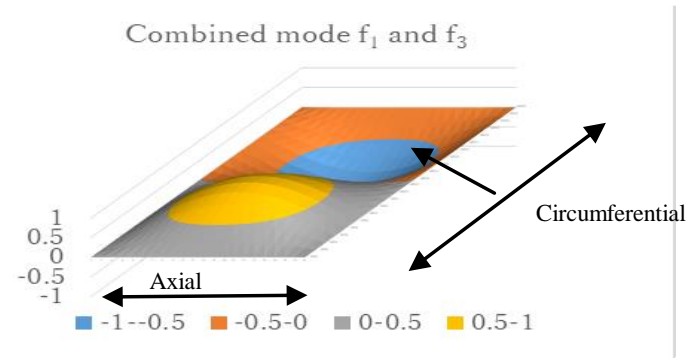

4th mode of circumferential direction

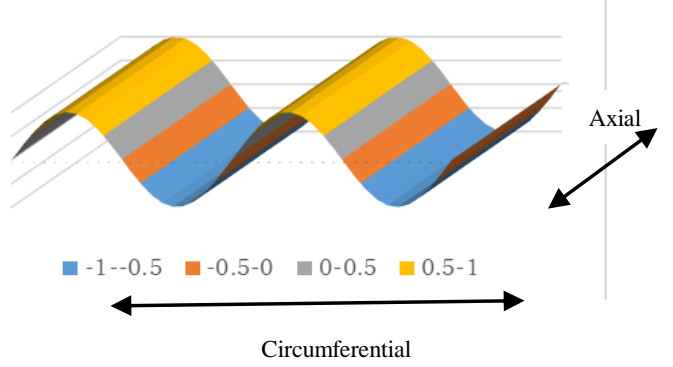

Fig.4 Equivalent of acoustic field 


\section{ESTIMATED BASIS}

\section{A. Vortex generation and Strouhal number}

Recently CFD (Computational Fluid Dynamics) has been widely used to examine the state of flow. Therefore, we try to use the CFD technique to obtain the state of flow and the Strouhal number. We performed the unsteady CFD analysis based on the two dimensional NS Equation here.

The computational grid was 176 (flow direction) $\times 61$ (height direction).

It is necessary to place a sufficient number of calculation grids in the boundary layer developing along the wall in order to grasp the separation phenomenon of the wall flow.

The thickness of this boundary layer can be estimated by $1 / R_{e}^{0.5}$ from the Reynolds number $R_{e}$ based on the inlet height length $H$. The present analysis $\left(R_{\mathrm{e}}=6 \times 10^{4}\right)$ took the minimum lattice width $\mathrm{O}\left(10^{-5}\right)$

The boundary condition of the velocity was given by $V_{\mathrm{x}}=10$ $\mathrm{m} / \mathrm{s}$ and $V_{\mathrm{y}}=0 \mathrm{~m} / \mathrm{s}$ at inlet, and $\partial V_{x} / \partial \mathrm{x}=\partial V_{y} / \partial \mathrm{x}=0 \mathrm{~m} / \mathrm{s}$ at outlet. The boundary condition on the wall is non slip $\left(V_{\mathrm{x}}=V_{\mathrm{y}}=0 \mathrm{~m} / \mathrm{s}\right)$. The pressure condition was imposed the Neumann condition $\frac{\partial p}{\partial n}=0$.

Where $\mathrm{n}$ is the normal vector to the boundary plane. And the calculation was performed by the unsteady analysis without the turbulent flow model. Fig.5(a) shows the grid geometry and Fig.5 (b) shows the state of flow at instant. From Fig.5 (b) it was confirmed the state of vortex shedding behind tubes. The frequency of this vortex shedding is $200 \mathrm{~Hz}$ as shown in Fig.6. The Strouhal number can be obtained as follows.

$$
S \mathrm{t}=f D / V_{\mathrm{g}}=200 \times 0.05 / 22.5=0.44
$$

$V_{\mathrm{g}}$ can be obtained by the law of continuous. That is to say, $H \cdot V=(H-D) \cdot V_{\mathrm{g}}, \mathrm{V}_{\mathrm{g}}=H /(H-D) \cdot V=90 /(90-50) \times 10=22.5$

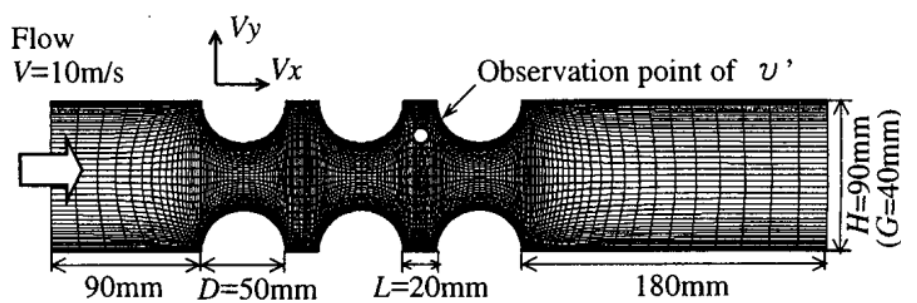

(a) Grid geometry

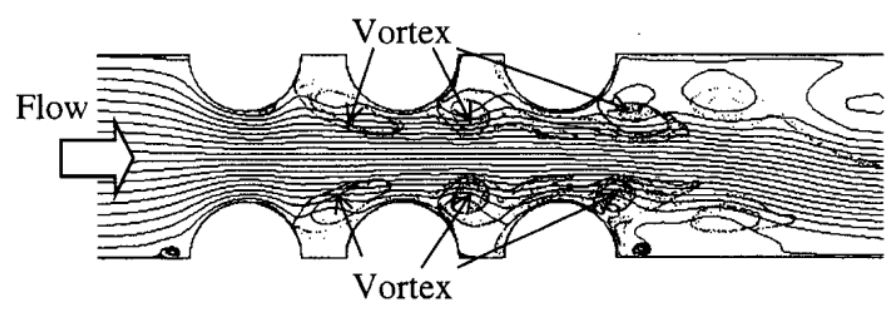

(b) state of flow at instant

Fig.5 Grid geometry and state of flow at instant

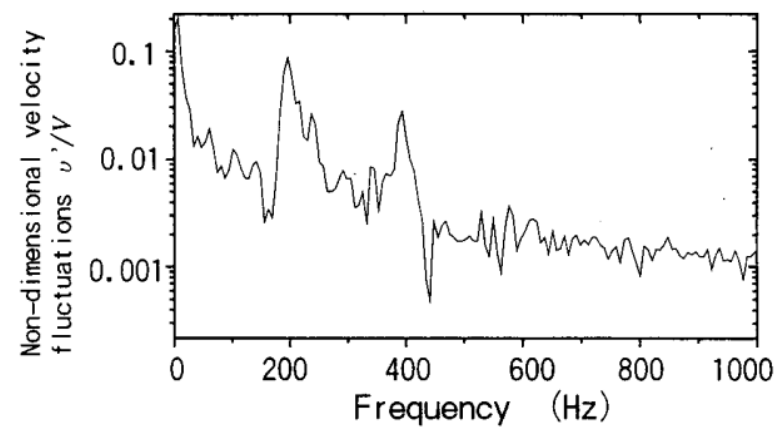

Fig.6 Spectrum of vortex shedding due to CFD

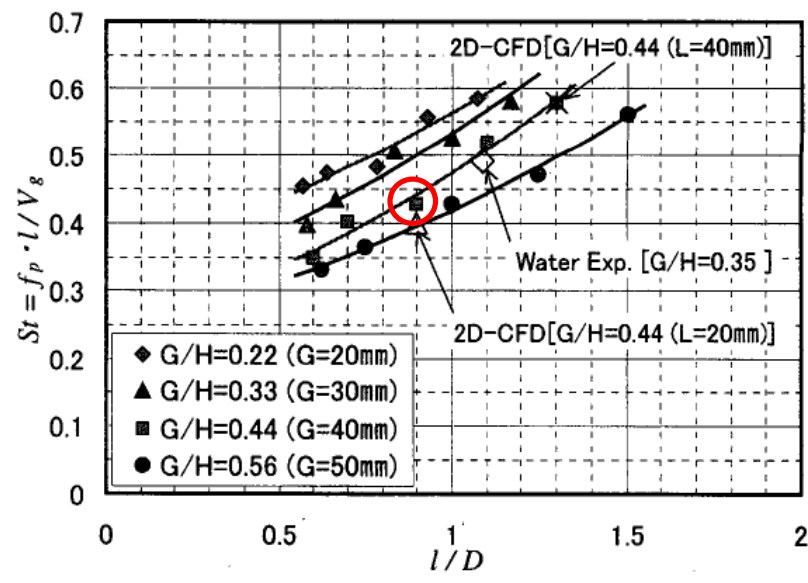

Fig.7 Strouhal number obtained by experiment

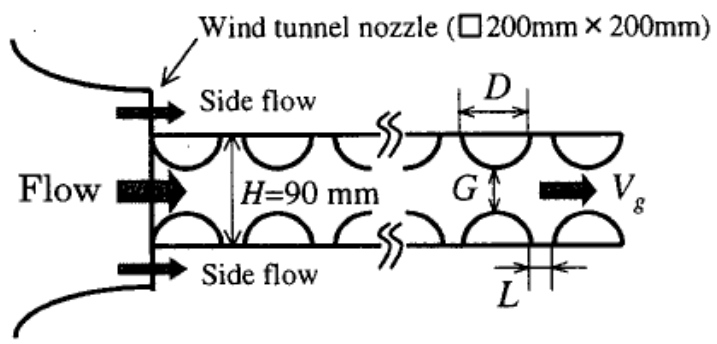

(a) Cross section

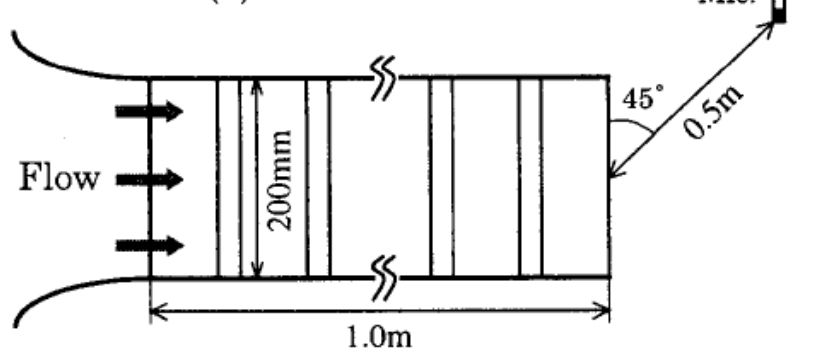

(b) Horizontal section

Fig.8 Schematic view of wind tunnel test apparatus

On the other hand, Aoki, et.al. obtained results shown in Fig.7 [16]. Here $G$ and $H$ are a gap length and a height length respectively as shown in Fig.8. And $l=L+D / 2$.

The present boiler, $l=45 \mathrm{~mm}$ as $D=50 \mathrm{~mm}, L=20 \mathrm{~mm}$, $G=40 \mathrm{~mm}, H=90 \mathrm{~mm}$. Therefore, the Strouhal number is about 0.43 from Fig.7 as $l / D=0.9, G / H=0.44$. This value 
coincides with that of the result due to CFD. Then I will examine results as $S t=0.44$ hereafter.

The vortex shedding frequency becomes

$f_{\mathrm{v}}=S \mathrm{t} \times V_{\mathrm{g}} / D=0.44 \times 13.5 / 0.05=119 \mathrm{~Hz}$ when the outlet velocity is $15 \mathrm{~m} / \mathrm{s}$, At that time the gap velocity becomes $V_{\mathrm{g}}=(9 / 5) \times 7.5=13.5 \mathrm{~m} / \mathrm{s}$

This value $119 \mathrm{~Hz}$ coincides with the natural acoustic frequency of $120 \mathrm{~Hz}$ which mode is combined mode of $f_{1}$ and $f_{3}$ described before.

\section{B. FEM Analysis Result of Resonance Mode}

The outline of the resonance frequencies and the corresponding modes were described in Chapter 2. In order to make it sure I carried out the FEM analysis.

The result is shown in Fig.9. From this figure, both resonance frequency and mode are the same as those of described in Chapter 2 It was confirmed that the presumed resonant frequency and mode are reasonable.

From this fact, the simple measurement which measures outside of the real boiler by a noise meter was clarified to be useful for the cause investigation.

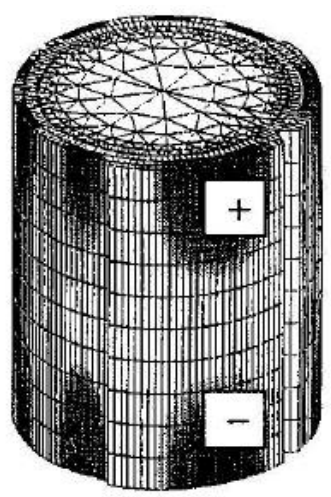

$120 \mathrm{~Hz}$



$160 \mathrm{~Hz}$
Fig.9 Modes of real boiler by FEM

\section{Effects of Each Countermeasure}

It was made clear by examination so far that the cause of abnormal tone was the resonance or self- excited tone by interference between the acoustic field and the vortex shedding. The following ways can be thought as coutermeasures.

(1) Add damping (Use of absorbing material)

(2) Weaken the strength of vortex which is exciting source

(3) Fix separating point not to cause lock-in

(4) Avoidance of resonance

It is impossible to perform the countermeasure (4) because the avoidance of resonance is a factor which gives effects to the performance by varying the velocity and the tube diameter. I will try to perform the countermeasures (2).

First the countermeasure (2) is explained as follows.

1) Attaching saw blade shown in Fig.10 to inlet of flow passage. Fig.10 shows two kinds of saw blade and the size is as of Fig.10. As a result, although not shown in the figure I can't almost obtain the countermeasure effect. But looking in detail, I can see the lowering of the sound pressure level and shifting of the occurrence velocity of abnormal tone higher. Therefore, this countermeasure is useful a little.

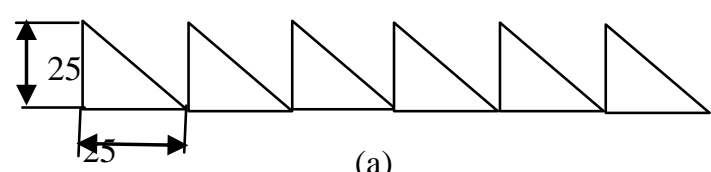

(a)

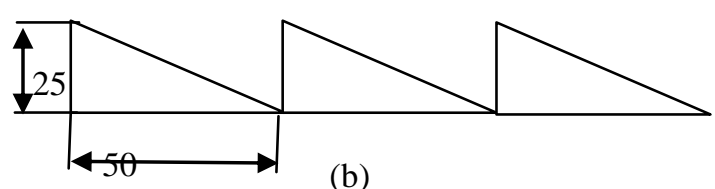

(b)

Fig.10 Configuration of saw blade

2) Making holes at plate connecting tubes

I will explain the effect of making holes at plate. I made holes of $\varphi 8$ with pitch $150 \mathrm{~mm}$ from $1^{\text {st }}$ row to $5^{\text {th }}$ row as shown in Fig.11. Fig.12 shows the result for cases of making holes. As can be seen from Fig.12 when the holes were pierced $1^{\text {st }}$ to $4^{\text {th }}$ row the sound pressure level became gradually lower and stop abnormal tone when the holes were pierced until $5^{\text {th }}$ row.

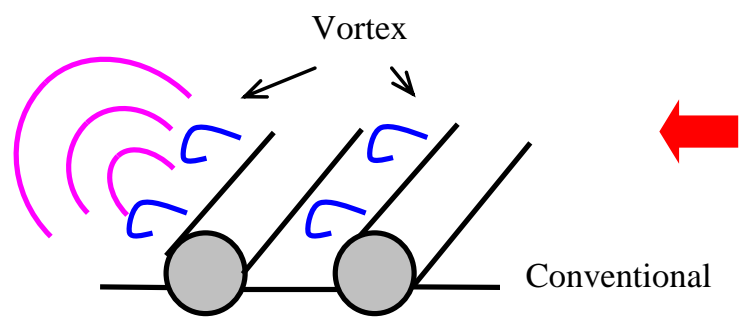

Through holes $\rightarrow$ Abnormal tone disappear

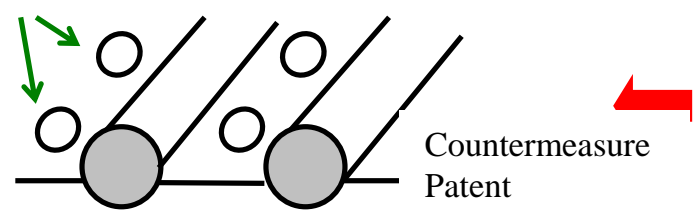

Fig.11 Outline of how to make through holes

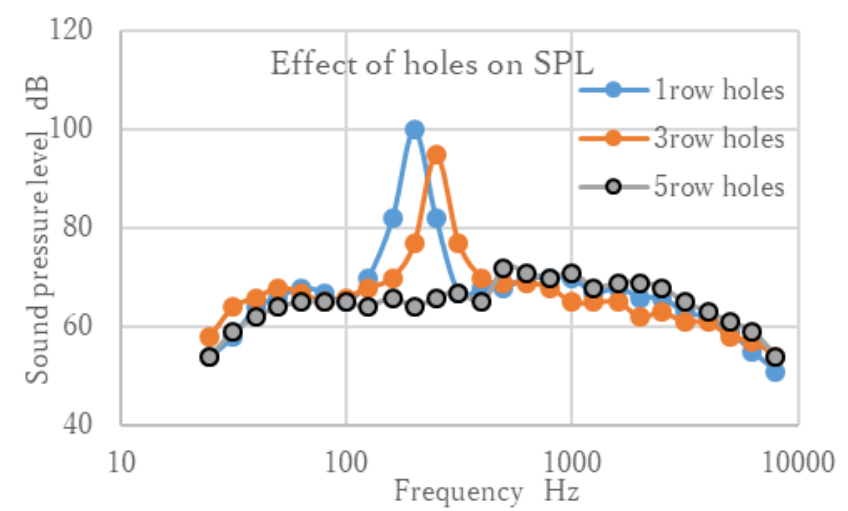

Fig.12 Effect of countermeasure on SPL 
From this experimental result, it is considered that when the hole is pierced the pressure difference between the inner furnace and the inner flow passage generates and the flux flowing into the flow passage destroys the vortex. As a result the vortex shedding is weakened and the abnormal tone disappeared. Moreover, I experienced that the self-sustained tone did not occur when the tube which was generator of vortex, was not put in the place the particle velocity was large, that is to say, neighborhood of the open end of the duct.

\section{CONCLUSIONS}

On the event of generating abnormal tone in a multi-tube type once-through boiler, research of causes on site was performed and showed their validities. As a result, the following findings could be obtained. That is to say,

The method piercing holes on a plate has a role destroying the periodic shedding vortex which is the exciting source and it is useful for the suppressing the abnormal tone. Moreover, the vortex generator like tubes should not put in the place where the particle velocity becomes large such as the end of the duct.

This paper shows strong on site color and not academically advanced. However, the unusual countermeasure making holes was successful for suppressing the abnormal tone and it is thought to be useful for the design engineers as the cases that worked.

\section{ACKNOWLEGEMENT}

I would deeply appreciate Dr Makoto Aoki who belongs The Kawasaki Heavy Industries, LTD. for me to write this paper. He measured the sound pressure level of the real once-through boiler on site and discussed the results.

\section{REFERENCES}

[1] R.C. Baird, "Pulsation-Induced Vibration in Utility Steam Generation Unit", Combust. 1954, 25, 38-44.

[2] P.R. Owen, "Buffeting Excitation of Boiler Tube Vibration," J. Mech. Eng. Sci. 1965, 7, 4, 431-439.

[3] Y.N. Chen, "Flow-induced Vibration and Noise in Tube-bank Heat Exchangers due to Von Karman Streets", J. Eng. Ind. 1968, 90, 1, 134-146.

[4] M. Funakawa, "The Acoustic Resonance in a Tube Bank". J. JSME. ,1970, 13, 348-355.

[5] J.A. Fitzpatric, "The Prediction of Flow-Induced Noise in Heat Exchanger Tube Arrays”, J. Sound. Vib., 1985, 99, 425-435.

[6] R.D. Blevins, Bressler M.M. "Acoustic Resonance in Heat Exchanger Tube Bundles-Part I: Physical Nature of the Phenomena", J. Press. Vess. Technol. , 1987, 10, 275-281.

[7] D.S. Weaver, "Vortex Shedding and Acoustic Resonance In Heat Exchanger Tube Arrays", Technology for the 90's, ASME Special Publication, New York, 1993; pp.776-810.

[8] S. Ziada, Oengören, A. "Vortex Shedding in an In-Line Tube Bundle with Large Tube Spacings", J. Fluid. Struct. , 1993, 7, 6, pp.661-687.

[9] S. Ziada, "Vorticity Shedding and Acoustic Resonance of Tube Bundles", J. Braz. Soc. Mech. Sci. 2006, 28, 2, pp.186-199.

[10] F. L. Eisinger and R. E. Sullivan, "Acoustic Resonance in a Package Boiler and Its Solution A Case Study", J. Press. Vess. Technol. 2006, 129, pp.759-762.

[11] H. Hamakawa, H. Matsue, E, Nishida and T. Fukano, "Acoustic Resonance and Vortex Shedding from Tube Banks of Boiler Plant", $J$. Fluid. Sci. Technol. 2008, 3, 6, pp.805-813.

[12] H. Hamakawa, T, Nakamura, K, Asakura, E. Nishida and E. Kurihara, "Effect of Arrangement of Tube Banks on Acoustic Resonance", O. J. Fluid. Dy. 2012, 2, pp.311-317.

[13] K. Ishihara, T. Tamehira, M. Tsujii and M. Ichimiya, "Study on a Countermeasure of Self-Sustained Tone by a Baffle Plate in Boiler Tube Banks", J. Basic. Appl. Phys. 2013, 2, Iss.3, pp.148-154.
[14] J. Kim, B. Cho, K. Kim, M.Y. Ha, C. Son and S.Y. Yoon, "Acoustic Resonance and Pressure Drop through a Staggered Tube Bundle in a Compact Heat Exchanger", Appl. Acoust. 2015, 91, pp.63-67.

[15] K. Ishihara and M. Nakaoka, "Study on a Countermeasure Using Walls Made of Perforated Plate for High Level Sound", In proceedings of ASME 2015 Pressure Vessels and Piping Conference Volume 4: Fluid-Structure Interaction, Boston, Massachusetts, USA, pp.19-23, July, 2015.

[16] M. Aoki, K. Ishihara and M. Koyama, "A Study on Aerodynamic Self-Sustained Tone Generated from a Multi tube Once-Through Boiler (Investigation of Generation Mechanism and Oscillating Frequency)", Transactions of the Japan Society of Mechanical Engineers, Series B, Vol.69, No.679(2003), pp.579-586(in Japanese).

Kunihiko Ishihara was born in 1947 in Kurashiki City, Okayama Prefecture Japan. He received the B.S. degree from Kobe University in 1969. He got a master's degree in Kobe University in 1971 and earned the Ph.D. degree in Engineering from The Osaka University in 1986.

He worked in Kawasaki Heavy Industry Co. Ltd. as an Mechanical Engineer for 33 years. After that he became a Professor of The University of Tokushima in 2004. He had been studying the vibration and noise control, above all he studied the flow induced vibration and noise problems. He has authored or co-authored over 100 technical journal and over 50 conference papers. He is a fellow of JSME (Japan Society of Mechanical Engineers) now. He is a Professor of Tokushima Bunri University. He teaches a mechanical field subjects for students. 